\title{
The vexing problem of post-amputation pain: What is the optimal perioperative pain management for below-knee amputation?
}

\author{
Patricia K. Morley-Forster, MD
}

Published online: 6 October 2009

(C) Canadian Anesthesiologists' Society 2009

Phantom pain, defined as intense throbbing or burning pain in the missing distal limb, is a common affliction after amputation. First described in 1872 by Ambroise Paré, the phenomenon is a common occurrence in $60-80 \%$ of amputees. ${ }^{1}$ Although some reports suggest abatement of phantom pain after a few years, others affirm it is longlasting. In addition to phantom pain, the unfortunate patient may also experience pain in the residual leg (stump pain) for reasons such as osteomyelitis, excessive scar tissue, or neuroma formation. In numerous pharmacotherapy trials, opioids, ketamine, anticonvulsants, and tricyclic antidepressants have provided only inconsistent relief.

Intense pre-amputation pain is likely to be associated with the development of long-term phantom pain. ${ }^{2}$ In the 1970s, this observation led researchers to hypothesize that pain created a permanent imprint in the dorsal horn and in the pain-processing regions of the central nervous system. A number of investigations were undertaken to reduce afferent input to the dorsal horn preoperatively and thus lessen the memory of pain in the central nervous system. The results of these trials, summarized below, have taught us a great deal about pain processing and have advanced knowledge in the field of regional analgesia. Nevertheless, pre-emptive analgesia yielded inconsistent results and does not appear to have made significant headway in reducing the vexing problem of long-term post-amputation pain.

The conclusion, based on new insights from animal and neuroimaging studies into the mechanism of phantom pain, is that reorganization in the somatosensory cortex is at least

P. K. Morley-Forster, MD ( $\square)$

Department of Anesthesia and Perioperative Medicine,

St. Joseph's Health Centre, University of Western Ontario,

268 Grosvenor St., London, ON N6A 4V2, Canada

e-mail: pat.morley-forster@sjhc.london.on.ca as important as events in the periphery. Reorganization may also occur at the dorsal horn and at the thalamic level and may be influenced by peripheral input. ${ }^{3}$

The mechanism of phantom limb pain remains obscure. It has the clinical characteristics of a neuropathic pain syndrome with a complex interplay between peripheral and central components, and it displays considerable variability among individuals. For example, when brachial plexus anesthesia was administered to prevent peripheral input from the amputation stump, both phantom pain and cortical reorganization were eliminated in $50 \%$ of upper-limb amputees, whereas the phantom pain remained unchanged in the remaining $50 \%$ of subjects. ${ }^{4}$

In order to attenuate the undesirable cortical reorganization, both sensory stimulation therapy and centrallyacting drugs, such as ketamine and gabapentin, have been proposed as preemptive therapies that could be used in the perioperative period for patients identified at high risk for phantom pain. ${ }^{3}$ What criteria should we use to determine those at highest risk? The observational study in this issue of the Journal is the first to suggest that pre-amputation use of prescribed opioids may be a risk factor for developing phantom pain. ${ }^{5}$

Since a landmark clinical trial published in 1988 by Bach et al., anesthesiologists have been interested in the role of perioperative neuraxial blockade as a pre-emptive treatment for phantom pain. Patients with vascular disease scheduled for amputation were randomized to receive either epidural bupivacaine for three days pre- and postprocedure or an epidural with placebo infusion plus conventional morphine analgesia postoperatively. ${ }^{6}$ After 6 months, the incidence of phantom limb pain was significantly lower in the epidural-treated group than in the morphine group. In 1994, this finding was confirmed in another randomized trial where an epidural was 
administered $48 \mathrm{hr}$ before amputation and continued until 72 hr postoperatively. $^{7}$

Since then, different modalities of pre-emptive analgesia have been explored. Although much has been learned about pain in the process, the clear, significant findings of the initial studies by Bach et al. ${ }^{6}$ and Jahangiri et al. ${ }^{7}$ on postamputation pain were not reproduced. It appears that shortterm preoperative blockade requires at least $48 \mathrm{hr}$ to be effective. Nikolajsen et $a .^{8}$ found that an epidural administered for only $18 \mathrm{hr}$ before lower extremity amputation had no effect on the incidence of phantom pain at 6 and 12 months postoperatively. His conclusions have influenced preoperative amputation protocols in anesthesia and surgical departments for many years.

To avoid the increased risks of neuraxial block, investigators began to utilize sciatic nerve sheath blocks placed perineurally by the surgeon at the time of amputation and continued for 48-72 hr. ${ }^{9}$ Lambert et al. ${ }^{10}$ compared epidural bupivacaine and morphine infusion $24 \mathrm{hr}$ preoperatively with continuous postoperative regional analgesia by directly-placed sciatic nerve sheath block in the popliteal fossa at the time of amputation. The epidural inserted preoperatively gave better pain relief in the immediate postoperative period but was no better than the perineural catheter at preventing phantom pain at 6 and 12 months postoperatively. Subsequent studies on lower extremity amputations utilizing peripheral nerve catheters have not shown a pre-emptive effect of peripheral nerve blockade though postoperative pain was well controlled. ${ }^{11}$

The intensity of both pre-amputation pain and acute postoperative pain is predictive of chronic pain development after lower extremity amputation. Which factor has the more significant effect? In a prospective study, Hanley et al. $^{12}$ attempted to untangle the contribution of preoperative pain $v s$ acute postoperative pain to the development of long-term phantom pain. Utilizing multiple regression analysis of a number of potential predictive risk factors, they confirmed the findings of others that pre-amputation pain intensity is the major predictor of acute phantom limb pain in the immediate postoperative period. Intense preamputation pain was correlated with more severe pain at postoperative days (POD) 4 and 5, but postoperative pain was not a significant predictor of phantom pain at any follow-up point. Anesthesiologists know how to control immediate postoperative pain; therefore, it is disappointing that aggressive pain management postoperatively does not appear to decrease persistent pain. Perhaps, as perioperative physicians, we should be casting our net more broadly and should be turning our attention to optimizing preoperative analgesia.

In this issue of the Journal, Roullet et al. report the results of an observational trial assessing the influence of chronic preoperative morphine use on the severity of post- amputation pain on 22 vasculopaths undergoing below knee amputation. A sciatic catheter was inserted in the popliteal fossa in all patients immediately before induction. Catheters were inserted easily with a nerve-stimulating catheter technique and all functioned successfully until their removal on POD 3. In addition, liberal intravenous morphine doses were offered until POD 3, at which time the patient was switched to regular subcutaneous and/or oral morphine supplemented by intravenous acetaminophen.

The patients were divided into two groups for statistical analysis. Patients were allocated to the No Preop Opioids group if they did not receive opioids preoperatively, while patients who were treated with preoperative opioids for more than 7 days were allocated to the Opioid Group. The two selected endpoints were the correlation of preoperative opioid dosing with postoperative opioid consumption on POD 3 and on POD 7. The last documented visit for the presence of phantom pain occurred on POD 7 , and the patients had their usual surgical follow-up visits for one year. At POD 3, the incidence of phantom limb pain was high and equivalent in both groups, $(>50 \%)$; however, it was significantly higher on POD 7 in the Opioid Group. Stump pain scores were higher on POD 1, 2, and 7 in the Opioid Group. Despite effective regional analgesia, morphine consumption from POD 1 to 3 and POD 7 was significantly greater in patients who had consumed opioids before surgery.

There were a number of limitations with this study design, the major one being that first contact with the patient was only one day prior to surgery. The preoperative opioid (oral morphine or a fentanyl patch) was not prescribed following predefined study criteria but according to the wishes of the doctor and the patient. The patient was only required to be on opioids for $>7$ days to be included in the Opioid Group, and the duration of opioid use was variable. All opioids were converted to intravenous morphine equivalents using standard equi-analgesic tables. Since these equivalents were established using data from patients on long-term opioids, their conversion to shortterm opioid doses may not have been accurate. Postoperative morphine consumption values were measured differently in some patients who were not placed on a patient-controlled analgesia (PCA) postoperatively due to cognitive deficiencies.

The authors raise the interesting question, "Are opioids themselves somehow responsible for the pain?" Given the observational nature of this study, there is not enough evidence to determine whether preoperative opioid use intensifies postoperative pain. It may well be that opioid use acted preoperatively as a surrogate marker for preoperative pain intensity. It is well known that intensity of phantom pain in the immediate postoperative period AND 
in the long term relates to the level of preoperative pain. Thus, the higher incidence of intense phantom pain in patients taking opioids preoperatively could have resulted from the more severe preoperative pain in these subjects. Support for this interpretation is presented by the preoperative difference in the mean visual analog scale (VAS) scores ( 2 in the No Opioids group vs 7 in the Opioid Group).

Patients in the opioid-treated group experienced more postoperative pain in the first two days and demonstrated higher VAS scores for stump pain despite functioning popliteal catheters. One possible explanation is that pain arising from the saphenous nerve territory was not covered; however, this would likely have had an equal effect on both groups.

In Seattle in 1995, Rapp et al. noted that standard regimens for PCA were less effective in opioid-tolerant patients on the Acute Pain Service. Those with preoperative exposure to opioids requested higher amounts from the PCA in the postoperative period to achieve the equivalent VAS scores. ${ }^{13}$ This confirmed an earlier observation by deLeon-Casasola et al. ${ }^{14}$ who found a three-fold increase in opioid requirements in those with prior opioid exposure for cancer pain treatments. The assumption was that the increase was due to tolerance to opioids, but in the past few years, the concept of opioid-induced hyperalgesia has gained increasing support. Paradoxically, sustained opioid administration in animal studies induces increased pain and tactile hypersensitivity or hyperalgesia. In response to regular intake of opioids, the neurotransmitters, cholecystokinin, and dynorphin A are secreted and facilitate pain signal transmission within the dorsal horn. ${ }^{15}$ The clinical significance of this for patients on long-term opioids has not been clarified. ${ }^{16}$

Roullet et al. have raised the question as to whether preoperative treatment with opioids ultimately contributes to more pain immediately after amputation and perhaps to a higher incidence of long-term pain. The observational study design has identified an association only and has not proven causation. In the belief that we can reduce the longterm incidence of phantom limb pain, should we withhold opiates and attempt to adhere to non-opioid analgesic protocols for preoperative pain management, even if the patient reports high preoperative pain? Unfortunately, this course of action may be deleterious, since it may promote high levels of preoperative pain that we know to be a risk factor for high postoperative pain.

Thanks to advances in regional analgesia, sciatic nerve catheters can be inserted in the popliteal fossa relatively easily with a very high success rate and with good postoperative pain relief for 2 to 3 days with additional rescue analgesics. If we are able to perform this technique, then we should. Neural blockade may help the patient to begin using the limb more quickly and may be a factor in an earlier prosthesis fitting. Early prosthesis use has been shown to reduce the emergence of phantom limb pain by reducing cortical reorganization patterns. ${ }^{17}$ When combined with an early and active physiotherapy program, this practice may be the way forward to improving function and modifying, if not eliminating, the devastating effects of phantom pain.

\section{Le problème épineux de la douleur post-amputation: Quelle est la prise en charge périopératoire optimale de la douleur lors d'une amputation tibiale?}

La douleur fantôme, définie comme une douleur pulsatile ou brûlante intense dans le membre distal manquant, est une affection courante à la suite d'une amputation. Décrite pour la première fois par Ambroise Paré en 1872, il s'agit d'un phénomène courant survenant chez 60 à $80 \%$ des amputés. ${ }^{1}$ Bien que certains comptes-rendus suggèrent qu'il y a diminution de la douleur fantôme après quelques années, d'autres affirment qu'elle perdure. Outre la douleur fantôme, le patient malchanceux pourrait également ressentir des douleurs dans la jambe résiduelle (douleurs de moignon) pour diverses raisons, comme par exemple l'ostéomyélite, une surabondance de tissu cicatriciel, ou la formation d'un névrome. Dans nombre d'études de pharmacothérapie, les opioïdes, la kétamine, les anticonvulsivants et les antidépresseurs tricycliques n'ont procuré qu'un soulagement variable.

Une douleur intense avant l'amputation sera probablement associée à l'apparition de douleurs fantômes à long terme. ${ }^{2}$ Dans les années 1970, cette observation a mené des chercheurs à poser l'hypothèse que la douleur créait une empreinte permanente dans la corne dorsale et dans les régions du système nerveux central impliquées dans le traitement des signaux nociceptifs. Plusieurs études ont été entreprises afin de réduire la contribution afférente de la corne dorsale en période préopératoire et ainsi réduire la mémoire de la douleur dans le système nerveux central. Les résultats de ces études, résumés ci-dessous, nous ont beaucoup appris sur les processus de traitement de la douleur et ont fait progressé les connaissances dans le domaine de l'analgésie régionale. Cependant, l'analgésie préventive n'a donné que des résultats inconstants et ne semble pas avoir fait beaucoup de progrès dans la réduction du problème épineux de la douleur post-amputation à long terme.

Sur la base de nouvelles perspectives tirées d'études animales et de neuro-imagerie portant sur les mécanismes 
sous-jacents des douleurs fantômes, il faut conclure que la réorganisation du cortex somatosensoriel est en tous cas aussi importante que les événements survenant en périphérie. Il est également possible qu'une réorganisation survienne dans la corne dorsale et au niveau du thalamus, laquelle pourrait être influencée par des apports périphériques. ${ }^{3}$

Le mécanisme induisant les douleurs fantômes dans les membres demeure obscur. Il possède les caractéristiques cliniques d'un syndrome de douleur neuropathique accompagné d'interactions complexes entre les composantes périphériques et centrales, et affiche une variabilité considérable d'un individu à l'autre. Par exemple, lors de l'administration d'une anesthésie du plexus brachial pour prévenir l'apport périphérique du moignon d'amputation, les douleurs fantômes et la réorganisation corticale ont toutes deux été éliminées chez $50 \%$ des amputés d'un membre supérieur, alors que la douleur fantôme est demeurée inchangée chez les $50 \%$ restants. $^{4}$

Afin d'atténuer la réorganisation corticale indésirable, deux traitements préventifs potentiellement utilisables dans la période périopératoire chez les patients identifiés comme présentant un risque élevé de manifester des douleurs fantômes ont été proposés : la thérapie par stimulation sensorielle et les médicaments à action centrale, tels la kétamine et la gabapentine. ${ }^{3}$ Quels sont les critères à utiliser afin de déterminer quels patients courent le risque le plus élevé? L'étude observationnelle présentée dans ce numéro du Journal est la première à suggérer que l'utilisation avant amputation d'opioïdes sous prescription pourrait constituer un facteur de risque d'apparition de douleur fantôme. ${ }^{5}$

Depuis la publication en 1988 d'une étude clinique phare par Bach et coll., les anesthésiologistes se sont intéressés au rôle des blocs neuraxiaux périopératoires en qualité de traitement préventif de la douleur fantôme. Des patients souffrant de maladies vasculaires et devant subir une amputation ont été randomisés à recevoir soit de la bupivacaïne péridurale durant trois jours avant et après l'intervention ou une perfusion péridurale de placebo ajoutée à l'analgésie conventionnelle avec morphine en postopératoire. ${ }^{6}$ Six mois plus tard, l'incidence de douleur fantôme était significativement plus basse dans le groupe traité par péridurale que dans le groupe traité à l'aide de morphine. En 1994, ces résultats ont été confirmés dans une autre étude randomisée, dans laquelle une péridurale avait été administrée $48 \mathrm{~h}$ avant l'amputation et maintenue pour $72 \mathrm{~h}$ après l'opération. ${ }^{7}$

Depuis, différentes modalités d'analgésie préventive ont été étudiées. Bien que nos connaissances de la douleur aient bénéficié de ces diverses explorations, les résultats clairs et significatifs des premières études de Bach et coll. ${ }^{6}$ et Jahangiri et coll. ${ }^{7}$ sur la douleur post-amputation n'ont pas été reproduits. Il semble qu'il faille au moins $48 \mathrm{~h}$ pour qu'un bloc préopératoire à court terme soit efficace. Nikolajsen et coll. ont découvert qu'une péridurale administrée durant $18 \mathrm{~h}$ seulement avant une amputation d'un membre inférieur n'avait pas d'effet sur l'incidence de douleur fantôme à six et douze mois après l'intervention. ${ }^{8}$ Leurs conclusions ont influencé pendant nombre d'années les protocoles préopératoires d'amputation dans les départements d'anesthésie et de chirurgie.

De façon à éviter les risques accrus du bloc neuraxial, les chercheurs ont commencé à utiliser des blocs de la gaine du nerf sciatique placés autour du nerf par le chirurgien au moment de l'amputation et maintenus pendant 48-72 h. ${ }^{9}$ Lambert et coll. ont comparé une perfusion péridurale de bupivacaïne et morphine $24 \mathrm{~h}$ avant l'opération à une analgésie régionale postopératoire continue procurée par un bloc de la gaine du nerf sciatique placé directement dans le creux poplité au moment de l'amputation. ${ }^{10}$ La péridurale placée avant l'intervention a permis un meilleur soulagement de la douleur dans la période postopératoire immédiate mais n'a pas été plus efficace que le cathéter sciatique pour prévenir les douleurs fantômes à 6 et 12 mois après l'intervention. Des études ultérieures portant sur des amputations des membres inférieurs et utilisant des cathéters des nerfs périphériques n'ont pas montré d'effet préventif des blocs du nerf périphérique, mais la douleur postopératoire a été bien contrôlée. ${ }^{11}$

L'intensité de la douleur avant amputation et le niveau de la douleur postopératoire aiguë sont des prédicteurs de manifestation de douleur chronique après l'amputation d'un membre inférieur. Quel facteur a l'effet le plus significatif? Dans une étude prospective, Hanley et coll. ont tenté de démêler la contribution de la douleur préopératoire par rapport à la douleur postopératoire aiguë dans l'apparition de douleur fantôme à long terme. ${ }^{12}$ À l'aide de l'analyse de régression multiple de plusieurs facteurs de risque potentiellement prédicteurs, ces auteurs ont confirmé les résultats d'autres chercheurs selon lesquels l'intensité de la douleur avant amputation est le prédicteur le plus important de douleur fantôme aiguë dans la période postopératoire immédiate. Une douleur avant amputation intense était corrélée avec une douleur plus forte durant les jours postopératoires 4 et 5 , mais la douleur postopératoire n'était pas un prédicteur significatif de douleur fantôme à quelque moment de suivi que ce soit. Les anesthésiologistes savent comment contrôler la douleur postopératoire immédiate; il est par conséquent décevant qu'une prise en charge énergique de la douleur en postopératoire ne semble pas diminuer les douleurs persistantes. Peut-être devrionsnous, en qualité de médecins spécialistes de la période périopératoire, élargir notre champ d'action et tourner notre attention vers d'autres modalités préopératoires, en optimisant par exemple les analgésiques oraux. 
Dans ce numéro du Journal, Roullet et coll. rapportent les résultats d'une étude observationnelle évaluant l'influence d'une utilisation chronique de morphine préopératoire sur la gravité de la douleur post-amputation chez 22 patients vasculopathes subissant une amputation tibiale. Un cathéter sciatique a été inséré dans le creux poplité immédiatement avant l'induction chez tous les patients. Les cathéters ont été aisément insérés à l'aide d'une technique par stimulation nerveuse et tous ont bien fonctionné jusqu'à leur retrait au jour postopératoire 3. En outre, des doses généreuses de morphine intraveineuse ont été proposées jusqu'au jour postopératoire 3, moment auquel elle a été remplacée par de la morphine sous-cutanée et/ou orale normale additionnée d'acétaminophène intraveineux.

Les patients étaient répartis en deux groupes pour l'analyse statistique. Les patients étaient alloués au groupe Sans opioïdes pré-op s'ils n'avaient pas reçu d'opioïdes en préopératoire, alors que les patients traités avec des opioïdes préopératoires pour plus de 7 jours ont constitué le groupe Opioïdes. Les deux critères d'évaluation choisis étaient la corrélation entre la dose d'opioïdes préopératoires et la consommation postopératoire d'opioïdes aux jours postopératoires 3 et 7. La dernière visite documentée portant sur la présence d'une douleur fantôme a eu lieu le jour postopératoire 7 , et les patients ont bénéficié des visites de suivi chirurgical habituelles durant un an. Au $3^{\mathrm{e}}$ jour postopératoire, l'incidence de douleur fantôme était élevée et équivalente dans les deux groupes (>50\%); toutefois, elle était significativement plus élevée au jour postopératoire 7 dans le groupe Opioïdes. Les scores de douleur de moignon étaient plus élevés les jours postopératoires 1, 2 et 7 dans le groupe Opioïdes. En dépit d'une analgésie régionale efficace, la consommation de morphine entre les jours postopératoires 1 et 3 et au jour postopératoire 7 était significativement plus élevée chez les patients ayant pris des opioïdes avant la chirurgie.

La méthodologie de cette étude comportait plusieurs limites, la principale étant que le premier contact avec le patient n'ait eu lieu qu'un jour avant la chirurgie. L'opioïde préopératoire (morphine orale ou timbre de fentanyl) n'a pas été prescrit selon des critères d'étude préétablis mais selon les désirs du médecin et du patient. Seuls les patients étant sous opioïdes pour $>7$ jours étaient inclus dans le groupe Opioïdes, et la durée d'utilisation des opioïdes était variable. Tous les opioïdes ont été convertis à des équivalents de morphine intraveineuse à l'aide de tables d'équipotence analgésique standard. Étant donné que ces équivalents avaient été calculés sur la base de données de patients sous opioïdes à long terme, leur conversion à des doses d'opioïdes à court terme pourrait n'avoir pas été précise. Les valeurs postopératoires de consommation de morphine ont été mesurées différemment chez certains patients ne recevant pas une analgésie contrôlée par le patient $(\mathrm{ACP})$ en postopératoire en raison de déficits cognitifs.

Les auteurs soulèvent une question intéressante : « Les opiö̈des en soi sont-ils d'une certaine manière responsables de cette douleur? » Étant donné le caractère observationnel de l'étude, nous ne disposons pas de données probantes suffisantes pour déterminer si l'utilisation préopératoire d'opioïdes intensifie la douleur postopératoire. Il se pourrait bien que l'utilisation d'opioïdes ait agi avant l'opération comme marqueur de substitution de l'intensité de la douleur préopératoire. Il est bien connu que l'intensité de la douleur fantôme dans la période postopératoire immédiate ET à long terme est liée au niveau de douleur avant l'amputation. Dès lors, l'incidence accrue de douleur fantôme intense chez les patients prenant des opiö̈des avant l'opération pourrait résulter en une douleur préopératoire plus prononcée chez ces patients. Cette interprétation est soutenue par la présentation des différences préopératoires en termes de scores moyens sur une échelle visuelle analogique (EVA) (2 dans le groupe Sans opiö̈des vs 7 dans le groupe Opiö̈des).

Les patients du groupe traité avec opioïdes ont ressenti davantage de douleur postopératoire au cours des deux premiers jours et ont eu des scores EVA de douleur de moignon malgré le bon fonctionnement des cathéters poplités. Une explication possible de ce phénomène est que la douleur provenant du territoire du nerf saphène interne n'était pas couverte; cependant, cela aurait probablement eu un effet semblable dans les deux groupes.

En 1995 à Seattle, Rapp et coll. ont remarqué que les régimes posologiques standard d'ACP étaient moins efficaces chez les patients tolérants aux opioïdes dans le Service de prise en charge de la douleur aiguë. Les patients exposés aux opioïdes avant l'opération nécessitaient des quantités plus importantes d'ACP en période postopératoire afin d'obtenir des scores EVA équivalents. ${ }^{13}$ Ces résultats sont venus confirmer une observation faite plus tôt par de LeonCasasola et coll., qui avaient noté une augmentation par trois des besoins en opioïdes chez les patients ayant été précédemment exposés aux opioïdes dans le cadre de traitements contre la douleur cancéreuse. ${ }^{14}$ L'hypothèse était que l'augmentation était due à une tolérance aux opiö̈des, mais au cours des dernières années le concept d'hyperalgésie induite par les opioïdes a fait son chemin et connaît désormais un soutien qui ne cesse de croître. Paradoxalement, l'administration soutenue d'opioïdes induit une douleur accrue et une hypersensibilité ou hyperalgésie tactile dans les études animales. En réaction à une prise régulière d'opioïdes, les neurotransmetteurs, la cholécystokinine et la dynorphine A sont sécrétés et facilitent la transmission du signal douloureux dans la corne dorsale. ${ }^{15}$ L'importance clinique de ce phénomène n'a pas été déterminée pour les patients sous opioïdes à long terme. ${ }^{16}$ 
Roullet et coll. ont soulevé la question de savoir si un traitement préopératoire à base d'opioïdes contribuait en fin de compte à davantage de douleur immédiatement après l'amputation et une incidence potentiellement plus élevée de douleur à long terme. La méthodologie de l'étude observationnelle n'a que souligné une association et n'a pas prouvé de lien de causalité. Afin d'agir sur notre conviction que nous pouvons réduire l'incidence à long terme des douleurs fantômes, devrions-nous suspendre l'administration d'opiacés et tenter d'adhérer à des protocoles analgésiques sans opioïdes pour la prise en charge de la douleur préopératoire et ce, même si le patient fait état d'une douleur préopératoire prononcée? Malheureusement, cette ligne de conduite pourrait s'avérer nocive, étant donné qu'elle pourrait promouvoir des niveaux élevés de douleur préopératoire, ce qui, nous le savons, constitue un facteur de risque de douleur postopératoire élevée.

Grâce aux progrès de l'analgésie régionale, les cathéters du nerf sciatique peuvent être placés relativement facilement dans le creux poplité avec un taux de réussite très élevé, et permettent un bon soulagement de la douleur postopératoire durant 2 ou 3 jours lorsqu'ils sont additionnés d'analgésiques de secours. $\mathrm{Si}$ nous sommes capables de réaliser cette technique, alors il faut le faire. Les blocs nerveux pourraient aider le patient à commencer à utiliser son membre plus rapidement et pourraient également jouer un rôle dans l'ajustement précoce d'une prothèse. Il a été démontré que l'utilisation précoce d'une prothèse réduisait l'apparition de douleur fantôme en réduisant les schémas de réorganisation corticale. ${ }^{17}$ Lorsque cette pratique est combinée à un programme précoce et actif de physiothérapie, elle pourrait être la voie à suivre pour améliorer le fonctionnement et modifier, voire éliminer, les effets dévastateurs de la douleur fantôme.

Competing interests None declared.

\section{References}

1. Nikolajsen L, Jensen TS. Phantom limb pain. Br J Anaesth 2001; 87: 107-16.

2. Nikolajsen L, Ilkjaer S, Kroner K, Christensen JH, Jensen TS. The influence of preamputation pain on postamputation stump and phantom pain. Pain 1997; 72: 393-405.
3. Flor H, Birbaumer N. Phantom limb pain: cortical plasticity and novel therapeutic approaches. Curr Opin Anaesthesiol 2000; 13 : $561-4$.

4. Birbaumer $N$, Lutzenberger $W$, Montoya $P$, et al. Effects of regional anesthesia on phantom limb pain are mirrored in changes in cortical reorganization. J Neurosci 1997; 17: 5503-8.

5. Roullet S, Nouette-Gaulain K, Biais M, et al. Preoperative opioid consumption increases morphine requirement after leg amputation. Can J Anesth 2009; 56(12).

6. Bach $S$, Noreng $M F$, Tjellden NU. Phantom limb pain in amputees during the first 12 months following limb amputation, after preoperative lumbar epidural blockade. Pain 1988; 33: 297-301.

7. Jahangiri M, Jayatunga AP, Bradley JW, Dark CH. Prevention of phantom pain after lower limb amputation by epidural infusion of diamorphine, clonidine, and bupivacaine. Ann R Coll Surg Engl 1994; 76: 324-6.

8. Nikolajsen L, Ilkjaer S, Christensen JH, Kroner K, Jensen TS. Randomised trial of epidural bupivacaine and morphine in prevention of stump and phantom pain in lower-limb amputation. Lancet 1997; 350: 1353-7.

9. Fisher A, Meller $Y$. Continuous postoperative regional analgesia by nerve sheath block for amputation surgery-a pilot study. Anesth Analg 1991; 72: 300-3.

10. Lambert AW, Dashfield AK, Cosgrove C, Wilkins DC, Walker AJ, Ashley $S$. Randomized prospective study comparing preoperative epidural and intraoperative perineural analgesia for the prevention of postoperative stump and phantom limb pain following major amputation. Reg Anesth Pain Med 2001; 26: 316-21.

11. Pinzur MS, Garla PG, Pluth T, Vrbos L. Continuous postoperative infusion of a regional anesthetic after an amputation of the lower extremity. J Bone Joint Surg Am 1996; 78: 1501-5.

12. Hanley MA, Jensen MP, Smith DG, Ehde DM, Edwards WT, Robinson LR. Preamputation pain and acute pain predict chronic pain after lower extremity amputation. J Pain 2007; 8: 102-9.

13. Rapp SE, Ready LB, Nessly $M L$. Acute pain management in patients with prior opioid consumption: a case-controlled retrospective review. Pain 1995; 61: 195-201.

14. deLeon-Casasola OA, Myers DP, Donaparthi S, et al. A comparison of postoperative epidural analgesia between patients with chronic cancer taking high doses of oral opioids versus opioidnaïve patients. Anesth Analg 1993; 7: 302-7.

15. Ossipov MH, Lai J, King T, Vanderah TW, Porreca F. Underlying mechanisms of pronociceptive consequences of prolonged morphine exposure. Biopolymers 2005; 80: 319-24.

16. Angst MS, Clark JD. Opioid-induced hyperalgesia: a qualitative systematic review. Anesthesiology 2006; 104: 570-87.

17. Lotze M, Grodd W, Birbaumer N, Erb M, Huse E, Flor H. Does use of a myoelectric prosthesis prevent cortical reorganization and phantom limb pain? Nat Neurosci 1999; 2: 501-2. 